\title{
Update on the utility of prognostic biomarkers in ovarian cancer
} Maurie Markman

\author{
Address: Department of Gynecologic Medical Oncology, The University of Texas, MD Anderson Cancer Center, 1515 Holcombe Boulevard, \\ Houston, TX 77005, USA \\ Email: mmarkman@mdanderson.org
}

FI000 Medicine Reports 2009, I:3I (doi: I0.34I0/MI-3I)

The electronic version of this article is the complete one and can be found at: http://www.Fl000.com/Reports/Medicine/content/I/3 I

\begin{abstract}
There is considerable interest in the gynecologic cancer community for the development of biomarkers that will be of utility in routine management of women with advanced ovarian cancer. However, at the present time, there remains limited evidence for the usefulness of any such test other than the serum cancer antigen (CA)-I 25 level, employed to monitor the course of disease in response to treatment or during periods of observation.
\end{abstract}

\section{Introduction and context}

Despite the high objective response rate (>60-70\%) of epithelial ovarian cancer to platinum-based cytotoxic chemotherapy, the majority of patients will ultimately experience progression of the disease process. Furthermore, as many as one-third of patients either will fail to experience any meaningful response to a frontline platinum-based regimen (disease progression or 'stable disease' as the best response) or will have very early documentation of progression (for example, during or within several months following the completion of primary therapy).

As a result, there has been considerable interest within the gynecologic oncology research community for the development of in vitro tumor assay systems or serum biomarkers that may be reliably used to (a) provide an accurate early indication of prognosis (prognostic test) or (b) to predict the effectiveness of an alternative management strategy in a patient whose disease has been documented to have progressed or where the test suggests an unfavorable outcome with the current strategy (predictive test). Unfortunately, despite considerable efforts by many investigators to establish reliable predictive tests, very few formally validated strategies (for example, estrogen receptor in breast cancer) currently exist in the oncology arena, and it would be most appropriate to firmly state that, to date, no such test satisfies this criterion in the area of epithelial ovarian cancer.

\section{Recent advances}

Conversely, a number of recent studies in ovarian cancer have reported on the ability of a variety of strategies to assist in the prognostic classification of patients with this malignancy. These approaches have included determining the relationship between the subtypes of infiltrating T-cells and other presumed immunoregulatory cells within the tumor $[1,2]$, specific (but quite complex) patterns of serum biomarkers observed prior to the administration of chemotherapy $[3,4]$, the unique methylation status of DNA obtained from the tumor [5], the presence (or absence) of a particular tumorassociated glycoprotein [6], specific pre-treatment cancer gene expression $[7,8]$, and mRNA profiles [9].

For a number of the newly reported prognostic factors, validation studies on a completely independent data set, undertaken by investigators not involved in the initial analysis, have yet to be performed (or at least have yet to be reported in the peer-reviewed oncology literature). Such efforts must be considered an absolute requirement before any new prognostic (or predictive) test can be seriously discussed for inclusion as a component within the routine management of ovarian (or any other type of) cancer. 
Although it would be appropriate to lodge criticisms similar to those noted above for the previously demonstrated prognostic utility of the serum cancer antigen (CA)-125 level, in a preliminary report of a phase 3 frontline ovarian cancer chemotherapy trial, a group of investigators proposed a rather provocative and novel use of this tumor marker [10]. The study compared an investigative approach (cisplatin plus topotecan followed by carboplatin plus paclitaxel) with a 'standard' carboplatin plus paclitaxel regimen, and surprisingly revealed a lower objective response rate and shorter progression-free survival associated with delivery of this experimental program.

One prospectively defined component of this trial was the collection of the serum CA-125 antigen level prior to the administration of the third cycle of chemotherapy. The study revealed a statistically significant $(P=0.006)$ lower percentage of patients in the experimental arm $(57.5 \%)$ whose serum antigen level had normalized by this point in the course of their illness, compared with the 'standard regimen' (66.3\%). Of relevance to the previous discussion of a predictive versus a prognostic test, the biological marker did not predict which patients would respond to a particular therapy but simply (and importantly) demonstrated that treatment on the control arm was associated with a superior prognosis.

\section{Implications for clinical practice}

In the opinion of this commentator, it is not unreasonable to inquire whether there is an actual need for any additional prognostic markers in advanced epithelial ovarian cancer beyond those currently routinely used (for example, cancer stage, tumor grade, optimal/ suboptimal surgical cytoreduction, patient age and existing serious co-morbidity, and in the setting of recurrent/persistent disease, prior therapy and treatment/ platinum-free interval).

Unless the knowledge gained through the completion of any of these tests is of substantially greater utility than tumor stage and grade (and so on) or the test can reproducibly predict a superior outcome if an alternative regimen (for example, 'drug $\mathrm{A}^{\prime}$ plus 'drug $\mathrm{B}^{\prime}$ rather than carboplatin plus paclitaxel) is employed based on the particular biological characteristics of the tumor, then what is the actual value associated with obtaining the test, outside the setting of an investigative trial? It will be important for investigators exploring the development of new prognostic markers in epithelial ovarian cancer to provide a response to this question.

Although the provocative results of the CA-125 study described above will need to be confirmed (validated) by investigators with independent data from separate trials, it may be possible to employ this objective, costeffective, and easily obtained 'marker of prognosis' to decide at a relatively early point in time whether a particular study should be closed for inferiority of one study arm or potential 'futility' (assuming no difference between the treatment regimens). Such an outcome may substantially shorten the duration of frontline ovarian cancer treatment trials and reduce the number of patients required to definitively document the effectiveness of a novel approach to management of the malignancy. Furthermore, strategies destined to be proven to be ineffective could be discarded, thereby minimizing the potential harm to research subjects who would otherwise have been treated with this investigative program.

It is also important to note the potential relevance of a focus on separately analyzing specific subtypes of ovarian cancer in future research efforts in this arena. For example, existing data demonstrate striking differences in the clinical behavior of mucinous and clear cell tumors of the ovary compared with that of other epithelial histologies [11]. In this regard, it may be possible to develop biomarkers that are highly clinically relevant for a particular subtype.

\section{Abbreviations}

CA-125, cancer antigen-125.

\section{Competing interests}

The author declares that he has no competing interests.

\section{References}

I. Bamias A, Koutsoukou V, Terpos E, Tsiatas ML, Liakos C, Tsitsilonis O, Rodolakis A, Voulgaris Z, Vlahos G, Papageorgiou T, Papatheodoridis G, Archimandritis A, Antsaklis A, Dimopoulos MA: Correlation of NK T-like $\mathrm{CD}^{+} \mathrm{CD}^{+} 6^{+}$cells and $\mathrm{CD}^{+} \mathrm{CD}^{2} 5^{+}$(hi) regulatory $\mathrm{T}$ cells with VEGF and TNFalpha in ascites from advanced ovarian cancer: association with platinum resistance and prognosis in patients receiving firstline, platinum-based chemotherapy. Gynecol Oncol 2008, 108:42|-7.

2. Sato E, Olson SH, Ahn J, Bundy B, Nishikawa H, Qian F, Jungbluth AA, Frosina D, Gnjatic S, Ambrosone C, Kepner J, Odunsi T, Ritter G, Lele S, Chen YT, Ohtani H, Old LJ, Odunsi K: Intraepithelial CD8 ${ }^{+}$ tumor-infiltrating lymphocytes and a high $\mathrm{CD}^{+} /$regulatory $T$ cell ratio are associated with favorable prognosis in ovarian cancer. Proc Natl Acad Sci U S A 2005, 102: 18538-43.

3. Zheng $Y$, Katsaros D, Shan SJ, de la Longrais IR, Porpiglia M, Scorilas A, Kim NW, Wolfert RL, Simon I, Li L, Feng Z, Diamandis EP: A multiparametric panel for ovarian cancer diagnosis, prognosis, and response to chemotherapy. Clin Cancer Res 2007, I 3:6984-92.

4. Oikonomopoulou K, Li L, Zheng Y, Simon I, Wolfert RL, Valik D, Nekulova M, Simickova M, Frgala T, Diamandis EP: Prediction of ovarian cancer prognosis and response to chemotherapy by a serum-based multiparametric biomarker panel. $\mathrm{Br} J$ Cancer 2008, 99:1 103-13. 
5. Barton CA, Hacker NF, Clark SJ, O'Brien PM: DNA methylation changes in ovarian cancer: implications for early diagnosis, prognosis and treatment. Gynecol Oncol 2008, 109:129-39.

6. Ponnusamy MP, Venkatraman G, Singh AP, Chauhan SC, Johansson SL, Jain M, Smith L, Davis JS, Remmenga SW, Batra SK: Expression of TAG-72 in ovarian cancer and its correlation with tumor stage and patient prognosis. Cancer Lett 2007, 25 I:247-57.

7. Birrer MJ, Johnson ME, Hao K, Wong KK, Park DC, Bell A, Welch WR, Berkowitz RS, Mok SC: Whole genome oligonucleotide-based array comparative genomic hybridization analysis identified fibroblast growth factor I as a prognostic marker for advanced-stage serous ovarian adenocarcinomas. J Clin Oncol 2007, 25:2281-7.

8. Jazaeri AA, Awtrey CS, Chandramouli GV, Chuang YE, Khan J, Sotiriou C, Aprelikova O, Yee CJ, Zorn KK, Birrer MJ, Barrett JC, Boyd J: Gene expression profiles associated with response to chemotherapy in epithelial ovarian cancers. Clin Cancer Res 2005, II:6300-10.
9. Merritt WM, Lin YG, Han LY, Kamat AA, Spannuth WA, Schmandt R, Urbauer D, Pennacchio LA, Cheng JF, Nick AM, Deavers MT, Mourad-Zeidan A, Wang H, Mueller P, Lenburg ME, Gray JW, Mok S, Birrer MJ, Lopez-Berestein G, Coleman RL, Bar-Eli M, Sood AK: Dicer, Drosha, and outcomes in patients with ovarian cancer. N Engl J Med 2008, 359:264I-50.

10. Hoskins PJ, Vergote I, Stuart G, Cervantes A, Tu D, Carey M, Provencher D, Katsaros D, Ghatage P, Eisenhauer EA: A phase III trial of cisplatin plus topotecan followed by paclitaxel plus carboplatin versus standard carboplatin plus paclitaxel as firstline chemotherapy in women with newly diagnosed advanced epithelial ovarian cancer (EOC) (OV.16). A Gynecologic Cancer Intergroup Study of the NCIC CTG, EORTC GCG, and GEICO. J Clin Oncol 2008, 26: 18S Part II: I0I Os. Abstract LBA5505.

II. Tian C, Markman M, Zaino R, Ozols RF, McGuire WP, Muggia FM, Rose PG, Spriggs D, Armstrong DK: CA-I25 change after chemotherapy in prediction of treatment outcome among advanced mucinous and clear cell epithelial ovarian cancers: a Gynecologic Oncology Group study. Cancer 2009, I I 5: I 395-403. 